\title{
Photodissociation dynamics of ionic argon pentamer.
}

\author{
Pavla Pukowiecová \\ Department of Physics, Faculty of Science, University of Ostrava, \\ 30. dubna 22, 70103 Ostrava, Czech Republic. \\ Florent Xavier Gadea* \\ LCPQ, IRSAMC and UMR5626 du CNRS, Université de Toulouse, \\ 118 route de Narbonne, 31062 Toulouse Cedex, France. \\ René Kalus ${ }^{\dagger}$ \\ Department of Applied Mathematics, \\ Faculty of Electrical Engineering and Computer Science, \\ VSB - Technical University of Ostrava, \\ 17. listopadu 15, 70833 Ostrava, Czech Republic.
}

(Dated: October 8, 2010)

\begin{abstract}
Photodissociation of the ionized argon pentamer, $\mathrm{Ar}_{5}^{+}$, is studied using an extended diatomicsin-molecules interaction model with the inclusion of the spin-orbit coupling and various dynamical approaches. A thorough comparison with the experimental data available in the literature is presented, including photofragment abundances and their kinetic and internal energy distributions. New predictions are reported for ultra-violet photoexcitation energies, a range that has not been studied before either experimentally or theoretically.
\end{abstract}

PACS numbers: 36.40.-c, 36.40.Wa, 36.40.Mr, 33.80.Gj

*Electronic address: gadea@irsamc.ups-tlse.fr

$\dagger^{\dagger}$ Electronic address: Rene.Kalus@vsb.cz 


\section{INTRODUCTION}

Ionic rare-gas clusters present various interesting particularities. As clusters, they are aimed to improve the understanding of the transition between the gas and the condensed phases of matter [1]. Although made of identical atoms, the charge is localized on a small chromophoric subunit [2-6], mainly trimer, but also tetramer or dimer, while the surrounding atoms are almost neutral. Therefore the rare-gas clusters are bound by heterogeneous interactions, like solvated species, with strong chemical interactions between the core atoms and loosely bound neutrals. However, the charge may migrate heavily, particularly after the clusters are excited. Reflecting their heterogeneity, when excited by photons or impacting electrons, they undergo an involved and rich non-adiabatic dynamics which is imprinted by a competition between the fission of the core, evaporation of neutrals, internal conversion, and relaxation over various electronic or nuclear degrees of freedom. Consequently, fast and slow, and neutral and charged fragments are produced. Moreover, the ionic rare-gas clusters can be easily produced via the adiabatic expansion through a nozzle and subsequent ionization, accelerated, size selected (thanks to their charge), and excited. The fragments can then be collected and analyzed in mass and velocity, or even in coincidence [7, 8]. Therefore, the ionic rare-gas clusters have been intensively studied for more than twenty years, both in experiment and in theory.

Almost all theoretical studies on the rare-gas ionic clusters rely on modeling with the diatomics-in-molecules approach [9] since it not only offers an efficient way for producing potential energy surfaces, as aimed initially [2], but also a realistic modeling of the electronic interactions [10]. Moreover, the method provides a diabatic electronic Hamiltonian which can be used, on the fly, in dynamical simulations [11, 12]. Based on the diatomics-in-molecules approach, a broad panoply of methods have been developed for the realistic simulation of photodissociation of rare-gas ionic clusters [13, 14]. They include sampling of the initial cluster configuration, modeling the photon absorption and the ensuing non-adiabatic dynamics spanning a broad manifold of electronic states as well as nuclear degrees of freedom, and analyzing the fragments. For example, the photodissociation of ionic rare-gas trimers has been thoroughly investigated [13-15] because the trimer is mainly the chromophoric core in larger clusters. In this case, only monomers are produced in experiments [16] with a bimodal kinetic energy distribution (fast and slow fragments) both for neutrals and for 
ions. Our earlier $[6,13,17-20]$ as well as recent $[14,15]$ theoretical simulations have nicely reproduced these experimental findings and showed that, in visible excitation, the trimer, initially almost linear and symmetric, mainly explodes leaving the central atom to rest in the center-of-mass. Interestingly, for the higher photon energies, the ions are produced in the high fine-structure state [15] corroborating the experimental findings [16]. It should be noticed that, in a very recent experiment on $\mathrm{Ar}_{2}^{+}$and $\mathrm{Ar}_{3}^{+}[8]$, the velocity vectors of all fragments have been collected in coincidence thanks to the development of sophisticated multicoincidence techniques. An additional interest of photodissociation studies is in that the initial state of the dynamics is well controlled by the photon absorption. The excitation energy is sharply determined and the absorption spectrum gives information on the electronic states populated and their weights.

The photodissociation of $\mathrm{Ar}_{5}^{+}$has been thoroughly investigated experimentally and interesting and detailed results have been reported for a whole range of visible excitation energies [21]. However, very few theoretical studies have been performed, maybe because of the large number of electronic states to be treated simultaneously, which makes this system challenging but difficult, particularly for the dynamics. Most importantly, the molecular dynamics simulation of such system requires the consideration of the coupling between electronic and nuclear motions. Hybrid approaches have been developed with a quantum treatment of electronic interactions coupled to classical trajectories for the nuclear degrees of freedom. Most of these methods are based on the mean-field approach where an effective potential energy surface is calculated on the fly through the Ehrenfest theorem [22]. Although this approach is very successful for the smallest clusters and leads to many valuable results, it finds limitations for the long-time dynamics because of producing fragments with fractional charges. According to the trajectory surface-hopping approach, another commonly used hybrid method proposed by Tully [23], the system evolves on a given adiabatic potential energy surface and quantum hops are proposed on the fly. Since a specific adiabatic state is followed at any time, there are no fractionally charged fragments, but the non-adiabatic couplings must be evaluated on the fly, in addition to the electronic Hamiltonian. We have recently proposed a novel method which combines mean-field and trajectory surface-hopping approaches and periodically introduces decoherence [24]. The application of this novel method to the photodissociation of $\mathrm{Ar}_{5}^{+}$, for which detailed experimental results are available, is an additional motivation to perform this study. 
The present contribution is organized as follows. In Sec. II, the methods and computational details are shortly recalled for consistency. Sec. III is devoted to our results on the $\mathrm{Ar}_{5}^{+}$cluster. The structures and thermodynamics of $\mathrm{Ar}_{5}^{+}$in the electronic ground state and its photoabsorption spectrum are at first discussed in Sec. III A since it is important to know the initial structures and electronic states from which the photodissociation of $\operatorname{Ar}_{5}^{+}$ starts at each specific photon energy. Then, results on the $\mathrm{Ar}_{5}^{+}$photodissociation, the core of the present work, are presented, discussed, and compared with the experiment in Sec. III B for the visible range of photon energies and some predictive simulations are reported for the photodissociation of $\mathrm{Ar}_{5}^{+}$in the ultra-violet band in Sec. III C. Finally, conclusive remarks are given in Sec. IV.

\section{METHODS AND COMPUTATIONS}

Any photodynamical calculation requires a few important ingredients: firstly, a reliable interaction model providing not only the electronic ground state of the system but also electronically excited states which are assumed to be involved at photon energies considered, secondly, a dynamical model to be used in calculations of the fragmentation dynamics ensuing the initial photoexcitation, and, last but not least, a model for a realistic description of transition probabilities for electronic jumps from the ground state to respective excited states. In this work, we use the methodology which was described at length in our previous studies and, consequently, only a brief summary of the methods is presented here. The reader is refered to the cited literature for more details.

The diatomics-in-molecules approach, invented originally by Ellison [9] and applied for the first time to rare-gas cluster cations by Kuntz and Valldorf [2] and extended by Amarouche et al. [3], is used to model the intra-cluster interactions in $\mathrm{Ar}_{5}^{+}$. The method consists in an expansion of the overall electronic Hamiltonian into a sum of diatomic and atomic terms [9],

$$
\hat{\mathrm{H}}=\sum_{P=1}^{N-1} \sum_{Q=P+1}^{N} \hat{\mathrm{H}}_{P Q}-(N-2) \sum_{P=1}^{N} \hat{\mathrm{H}}_{P}
$$

and designing an appropriate basis set of electronic wave functions which would allow to construct the corresponding Hamiltonian matrix in terms of only atomic and diatomic energies. For an $N$-atom ionic rare-gas cluster, $\operatorname{Rg}_{N}^{+}$, Kuntz and Valldorf [2] proposed to use a minimal basis set of $3 N$ valence bond Slater determinants, each representing one of the 
approximately diabatic electronic states with the positive charge localized on a particular atom, $K=1, \ldots, N$, in a $p_{m}$ orbital, $m=\mathrm{x}, \mathrm{y}, \mathrm{z}$. We denote this simple model as DIM in this work. Amarouche et al. [3] extended this simple model by including the spin-orbit interaction via the atoms-in-molecules approach [25], which required a) doubling the basis set by considering two possible orientations of the spin of the electron removed from a particular $p$-orbital (localized on a particular rare-gas atom), $s_{z}= \pm 1 / 2$, and b) amending the atomic terms of Eq. 1 with atomic spin-orbit Hamiltonians for $\mathrm{Rg}^{+}$. We denote this extended model as DIM+SO throughout this study. As we found in a previous work [14], the spin-orbit coupling may be important even for argon if subtle features of the photodynamics are considered. Consequently, the extended DIM+SO model is predominantly used in this work. The simpler DIM model has been called-up only in a few calculations to assess the importance of the spin-orbit coupling in $\mathrm{Ar}_{5}^{+}$for the processes considered in this work. A couple of additional corrections can further be included in the original DIM model, namely the leading polarization [3] and dispersion [26] three-body forces, which advance the DIM model beyond the purely pair-wise additive level. Since such corrections do not seem important for small ionic clusters of argon [6], they are not considered here to reduce computational demands [27]. In the extended DIM+SO model, the electronic Hamiltonian matrix of an $\operatorname{Rg}_{N}^{+}$cluster is a $6 N \times 6 N$ matrix $\left(30 \times 30\right.$ for $\left.\operatorname{Ar}_{5}^{+}\right)$and the lowest $3 N\left(15\right.$ for $\left.\operatorname{Ar}_{5}^{+}\right)$ doubly degenerate electronic states can be obtained by diagonalizing it. These states cover entirely both the visible (VIS) and ultra-violet (UV) range of photon energies and are thus sufficient for modeling the photoabsorption spectrum and the photodissociation dynamics of $\mathrm{Rg}_{N}^{+}$in both the VIS and UV part of the electromagnetic spectrum. The DIM+SO model requires a few inputs which have to be taken from independent sources, namely the potential energy curves for the electronic ground state and three lowest excited states of ionic $\operatorname{Rg}_{2}^{+}$, the electronic ground-state potential for neutral $\mathrm{Rg}_{2}$, and the spin-orbit coupling constant for $p$ electrons of atomic ions $\mathrm{Rg}^{+}$. In this work we use the diatomic curves for $\mathrm{Ar}_{2}^{+}$taken from ab initio calculations [28], a state-of-the-art semiempirical potential for neutral $\mathrm{Ar}_{2}$ [29], and the spin-orbit coupling constant extracted from experimental data on the splitting between the ${ }^{2} P_{1 / 2}$ and ${ }^{2} P_{3 / 2}$ fine-structure states of $\mathrm{Ar}^{+}$[30]. The DIM+SO model used in this work has many times been tested successfully against available experimental data on small and medium-size argon clusters, including their structures and energetics [6, 31], photoabsorption [6], and non-adiabatic dynamics [14, 15, 32], and can, thus, be considered 
well established for the description of intra-cluster interactions in these clusters.

The dynamical methods we use in this work to propagate photoexcited $\mathrm{Ar}_{5}^{+}$in time are semi-classical and are based on the Ehrenfest, mean-field approach, i.e., classical equations of motion are used for heavy nuclei moving in a mean-field potential generated by quantum electrons,

$$
\dot{Q}_{I}=\frac{P_{I}}{M_{I}}, \quad \dot{P}_{I}=\left\langle\psi\left|-\frac{\partial \hat{\mathrm{H}}}{\partial Q_{I}}\right| \psi\right\rangle,
$$

and quantum, time-dependent Schrödinger equation is employed for propagating the electronic wave function in time,

$$
i \hbar \frac{\partial|\psi\rangle}{\partial t}=\hat{\mathrm{H}}|\psi\rangle
$$

This approach was coupled with the DIM interaction model for $\mathrm{Ar}_{N}^{+}$clusters in early works of one of us, the HWD model of Refs. 10-12, and extended recently [32] by the inclusion of the spin-orbit interaction. A detailed overview of the equations of motions, Eqs. 2 and 3 , rewritten in matrix form as well as details on the implementation of these equations are given in Ref. 32. In accordance with our previous studies, we denote this dynamical model as MF (Mean-Field) throughout this work. Very recently [24], we extended the MF model by including quantum decoherence via periodically collapsing the electronic wavefunction to one of the adiabatic electronic states available within the diatomics-in-molecules model. As shown in Ref. 24, the inclusion of quantum decoherence in the MF model leads to a substantial improvement of our theoretical data on the post-ionization fragmentation of rare-gas trimers. We denoted this improved dynamical model as MFQ (Mean-Field with Quenchings) and developed several semi-empirical schemes for the inclusion of quantum decoherence to the original MF method. Each of these schemes consists of two steps: a) invoking an instantaneous collapse of the electronic wavefunction (quenching) and b) adjusting nuclear velocities so that the overall energy be conserved. Two algorithms were used for step (a). In the AMP method of ref. 24, the collapse probabilities are obtained from electronic wavefunction amplitudes calculated for the current (nuclear configuration dependent) adiabatic basis set; in the TFS method, the Tully's fewest switches algorithm [23] is used for this purpose. Concerning the second step, b, the nuclear velocities are adjusted either via scaling by a scalar factor, the S method of Ref. 24, by adding a vector parallel to the real part of the non-adiabatic coupling vector as proposed originally by Tully [23], method $\mathrm{C}$, or adding a vector parallel to the gradient of the difference of adiabatic electronic 
energies corresponding to the states the system jumps from and to [33], respectively, method G. The two methods for calculating the collapse probabilities, step a, and the three methods for the after-collapse adjustment of nuclear velocities, step b, give rise to six different schemes for the inclusion of quantum decoherence processes at the MFQ level (see Ref. 24 for details). In this work, we use two of them: a) the method combining the AMP approach for calculating the collapse probabilities and the $\mathrm{S}$ algorithm for adjusting nuclear velocities (following the nomenclature of Ref. 24, we denote this method as MFQ-AMP/S), and b) another method combining the TFS and G approaches (hereafter denoted as MFQ-TFS/G). The former method is computationally cheaper and is used as the main dynamical tool in this work, the latter method is used to provide comparative numerical data. Note also that the MFQ methods remove a principal insufficiency of the original MF model, namely partial electric charges carried by photofragments, which sometimes confuses the evaluation and interpretation of numerical data. Employing the MFQ approaches has allowed us, for example, to see the internal states (either electronic or rovibronic) of the photofragments in this work and to analyze their stability, which would have been impossible within the original MF model.

In the experiment, the photodissociation data are not measured on a single molecule or a cluster. Instead, averaged signals are recorded from macroscopic ensembles of species, the initial, post-excitation states of which are different. In theory, this is usually modelled by calculating bunches of trajectories corresponding to different initial conditions generated for each photon energy and subsequent averaging calculated numerical data. Within a semiclassical approach, the initial conditions consist of the initial positions and velocities of nuclei at the instant of the photon absorption and the initial state of electronic cloud (amplitudes of the adiabatic electronic states populated after absorbing a photon of a predefined energy). In this work, 500 trajectories have been employed for each particular photon energy, the initial conditions of which have been generated in two steps (see also Ref. 14 for more details). Firstly, a large set of configurations of $\mathrm{Ar}_{5}^{+}$has been generated using the constanttemperature Monte Carlo method. Several temperatures have been used in test calculations leading to fairly similar results and, consequently, most of the present work has finally been done for a single temperature, $T=50 \mathrm{~K}$. The initial velocities of the nuclei have been set to zero as thermal energies corresponding to the temperatures considered are negligible as compared to both VIS and UV photon energies. About $20 \times 10^{6}-40 \times 10^{6}$ of $\operatorname{Ar}_{5}^{+}$ 
configurations have been generated for each photon energy in these Monte Carlo calculations from which only every 200th configuration have been used in further processing to avoid unphysical correlations. As a result, a file of 100,000 - 200,000 $\mathrm{Ar}_{5}^{+}$configurations has been created. Secondly, after this large ensemble of configurations has been created, $500 \mathrm{Ar}_{5}^{+}$ configurations have randomly been transfered from it to the final set of initial conditions according to the electronic transition probabilities. These probabilities have been calculated for each cluster configuration within the first-order perturbation theory via the square of the transition dipole moment between the electronic ground state and the excited state the energy difference of which from the ground state fits in a $0.05 \mathrm{eV}$ window around the specific photon energy. We use the same methodology for the transition dipole moment calculations as that developed previously and tested in numerous photoabsorption modelings on raregas cluster cations, including argon $[6,14]$. The methodology is based on the point-charge approximation [34] combined with the inclusion of atomic polarization effects [35]. For details see Ref. 36.

A detailed description of the numerical implementation of the MF and MFQ methods can be found in Refs. 32 and 24, respectively. Here, we recall only briefly some technical items pertinent to the present work. For each trajectory, equations of motion 2 and 3 have been solved numerically for a 50 ps time interval using a Runge-Kutta $4^{\text {th }}$-order method with the time step set to $0.25 \mathrm{fs}$. At $t=50 \mathrm{ps}$, the integration has been stopped and fragments have been analyzed. Since the photodissociation is a rather fast process due to a large amount of energy pumped into the cluster by the absorbed photon, such an integration time is sufficient for most of the photodissociation trajectories. However, for selected photon energies, the integration of Eqs. 2 and 3 has been extended to 200 ps to see the stability of photofragments detected at $t=50 \mathrm{ps}$. The period for collapsing (quenching) the electronic wavefunction in the MFQ calculations have been chosen in accordance with our previous study [24], $\Delta t_{\mathrm{Q}}=100 \mathrm{fs}$ for the MFQ-AMP/S method and $\Delta t_{\mathrm{Q}}=10 \mathrm{fs}$ for the MFQ-TFS $/ \mathrm{G}$ method. Moreover, several quenching periods have been tested for selected photon energies and no remarkable dependence of the results on the quenching period has been observed. 


\section{RESULTS AND DISCUSSIONS}

\section{A. Structures, thermodynamics, and absorption spectrum of $\mathbf{A r}_{5}^{+}$}

Before absorbing a photon, the cluster is in its electronic ground state. It is important to know its possible structures and have an idea of their distribution. The stable and metastable structures of $\mathrm{Ar}_{5}^{+}$we obtained for the DIM+SO model are summarized in Fig. 1. The most stable structure of $\mathrm{Ar}_{5}^{+}$consists of an almost linear and only slightly asymmetric trimer core, with about half the charge deposited on the central atom with the two outer atoms sharing the remaining half, and two outer, almost neutral atoms in a crown around one of the ionic bonds. A similar structure, a strictly symmetric and almost linear ionic core with the two neutral atoms attached aside, but in different crowns, is only by $+2 \mathrm{meV}$ higher than the most stable structure. At higher energies, a linear tetramer core can be formed (+28 meV above the most stable structure), and at much higher energies a dimer-core structure $(+83 \mathrm{meV})$ or even a linear centrosymmetric arrangement $(+95 \mathrm{meV})$ appear. Up to now, only the potential energy has been considered and we are therefore implicitly in the microcanonical ensemble. When considering the canonical ensemble, there are distributions of allowed potential energies and therefore of structures, and thermodynamical quantities should be considered. This is illustrated by the evolution with temperature for various thermodynamical and structural parameters reported in Fig. 2. The heat capacity of $\mathrm{Ar}_{5}^{+}$ (more precisely, the interaction part of the heat capacity in which the constant kinetic energy contribution is not considered) varies only slightly with $T$ below the temperature region in which the evaporation of monomers becomes important (see Fig. 2a). However, the increase of the heat capacity becomes sharp at $T \geq 90 \mathrm{~K}$ indicating some pre-evaporation processes in $\mathrm{Ar}_{5}^{+}$at these temperatures. A small peak, which appears at $80 \mathrm{~K}$, should be probably related to melting in the neutral crowns around the trimer core represented by an increased angular mobility of the neutral atoms. The evolution of the average distance of atoms from the cluster center-of-mass (black points in Fig. 2b), confirms this conclusion. In contrast to what is observed for the neutral atoms, the three ionic core atoms remain close together for all the temperatures considered (even above the evaporation limits), and their average distance from the cluster ionic core center-of-mass (empty circles in Fig. 2b) even slightly decreases at high temperatures, mainly due to bending. The increase in the global average 
distance from the cluster center-of-mass is thus clearly due to the two neutral, loosely bound atoms, the mobility of which increases strongly after $T=110 \mathrm{~K}$ even in the radial direction. The third parameter considered in Fig. 2, $\Delta Q \equiv Q_{1}-Q_{2}+Q_{3}-Q_{4}+Q_{5}$ [37], but effectively $\Delta Q \approx Q_{1}-Q_{2}+Q_{3}$, is used to assess the charge mobility over the cluster. It is clear that $\Delta Q=0.5$ for the linear centrosymmetric ionic trimer core [38], while $\Delta Q=0.0$ for the positive charge localized on a centrosymmetric tetramer or on a dimer. As the temperature increases, we see in panel (c) of Fig. 2 a small but regular decrease of this parameter. It indicates a growth of the positive charge fluctuations, mainly within the trimer core, but with a small increase of the abundance of tetramer-core structures at higher temperatures (see also Fig. 3a). This thermodynamical analysis consistently shows that the cluster core remains mostly trimeric as the temperature rises, while the two outer atoms melt and become mobile. The small amount of the tetramer-core structures appearing at higher temperatures need not be considered, as they are eventually filtered off by the selection rules for electronic excitations ( $c f$. Fig. 3b and the discussion below in this sub-section).

The photoabsorption spectrum of $\mathrm{Ar}_{5}^{+}$, as obtained from our calculations, is reported in Fig. 4. In panel (a), total photoabsorption cross sections are depicted for a broad range of photon energies, various temperatures, and interaction models; in panel (b), the total photoabsorption spectrum (thick gray curve) is accompanied by partial absorption profiles obtained for particular transitions from the electronic ground state of $\mathrm{Ar}_{5}^{+}$to respective excited states (black curves). Panel (b) documents thus how different electronic transitions contribute to the overall photoabsorption of the $\mathrm{Ar}_{5}^{+}$cluster. The photoabsorption curves have been computed, as in our previous studies [6, 36, 39], assuming vertical electronic transitions and accumulating a quantity proportional to the square of the electric transition dipole moment at geometries sampled at a given temperature by the means of the canonical Monte Carlo algorithm. The absorption spectra of $\mathrm{Ar}_{5}^{+}$do not depend much on the interaction model used. As shown in the inset of Fig. 4a, including the spin-orbit coupling or induced dipole - induced dipole interactions leads to only minor changes in the photoabsorption profiles. The $\mathrm{Ar}_{5}^{+}$spectrum looks like the one of the trimer, with a strong VIS peak and two red and blue shifted wings, and a weaker band in the UV. This similarity clearly emphasizes that the trimer core is effectively the chromophore in the pentamer and absorbs the photon. However, there are some differences in the charge distributions in the absorbing excited states, which correspond to the initial states for the photodissociation dynamics. See 
Table I for a detailed summary on the positive charge delocalization in the electronically excited states for the presumingly two most important structural isomers of $\mathrm{Ar}_{5}^{+}$. For the trimer cation, the charge is localized on the two outer atoms after the excitation in the main VIS band, while now, for the pentamer, considerable part of the positive charge is transfered to the two originally neutral, crown atoms (see, e.g., states $4-12$ of Table I contributing to the main $\mathrm{Ar}_{5}^{+}$photoabsorption peak, or states 2 and 3, and 13 and 14 contributing to the absorption wings observed in the VIS part of the photoabsorption spectrum of $\mathrm{Ar}_{5}^{+}$). Only for the UV range, the charge delocalization in the respective excited state of $\mathrm{Ar}_{5}^{+}$(state 15) resembles closely what we observe in the ionic trimer. When varying the temperature, there is some decrease in the main VIS band while the UV one becomes more intense and wider. Unfortunately, there is no experimental absorption spectrum reported for $\mathrm{Ar}_{5}^{+}$in literature to compare with and to be used in an estimation of the temperature of experimental clusters (as was done in one of our previous studies on the ionic trimer of argon [37]). For example, we obtained the best agreement of the VIS photoabsorption profile of $\mathrm{Ar}_{3}^{+}$with experimental data [40] for $T \approx 150 \mathrm{~K}$ [41]. Considering strong bonds in the ionic trimer of argon, compared to only loose bonding of the neutral atoms in $\mathrm{Ar}_{5}^{+}$, we would estimate, that the effective temperature of experimental populations of the ionic argon pentamer is considerably lower than that reported for the trimer.

As discussed in Sec. II, the initial configurations of $\mathrm{Ar}_{5}^{+}$, to be propagated in time during the dynamical part of calculations, have been generated in two steps. First, a thermal population of argon pentamer cations has been assembled for a pre-defined temperature (mostly $T=50 \mathrm{~K}$ in this work) using a canonical Monte Carlo algorithm, then, a smaller subpopulation has been selected for each photon energy according to the excitation probability calculated as described in Sec. II. The abundances of various isomers of $\mathrm{Ar}_{5}^{+}$in a purely thermal population are depicted in panel (a) of Fig. 3 and have been discussed above in this section. However, a natural question arises, how these thermal abundances are changed after invoking the excitation probability re-weighting. Illustrative results of such a re-weighting performed for various photon energies and for $T=50 \mathrm{~K}$ are shown in panel (b) of Fig. 3 . Clearly, the thermal abundances of the three lowest isomers of $\mathrm{Ar}_{5}^{+}$are more or less preserved after the excitation probability re-weighting with only minor, but systematic changes: a) the abundance of the most stable isomer of $\mathrm{Ar}_{5}^{+}$, isomer 1 of Fig. 1, is slightly enhanced with respect to the thermal population for essentially all the photon energies considered and 
b) the reverse holds for isomer 2. The abundance of the tetramer-core isomer, isomer 3 , is practically zero for all the photon energies considered, except for a very slight enhancement seen at the lowest energies included. Such an enhancement compares well with the fact that the tetramer-core isomers have their photoabsorption spectrum shifted considerably to the red and infra-red region of photon energies [39].

\section{B. Photodynamics in the main (VIS) absorption band}

After absorbing a photon, the excited cluster evolves on a manifold of excited states and since the excitation energy is rather high, it fragments. For the trimer and visible excitation, calculations have shown [12-14] that the dynamics is mainly explosive following essentially a symmetric elongation of the cluster, and the charge, initially shared by the outer atoms, is distributed among the fragments in the near asymptotic region. This simple picture nicely explains the experimental bimodal kinetic energy distribution (KED) [16, 42, 43] found for the ions as well as for the neutral monomer fragments, and also the relative height of the fast and slow peaks in both distributions. Now, for $\mathrm{Ar}_{5}^{+}$, the situation is more involved. On one side, we could expect that since the trimer is still the chromophore, similar dynamics should occur. However, as shown in the previous section, the excited states now often involve the atoms in the crown, which are neutral before the photon absorption. Therefore they could also play a role in the dynamics instead of remaining simple spectators of the trimer core explosion. Experimentally, the evolution of the KED of the ionic fragments with the photon energy, either monomers or dimers (no larger fragments were found experimentally), has been measured [21] in the range of $2.1-2.7 \mathrm{eV}(470-580 \mathrm{~nm})$, i.e., in the main visible excitation band. Fragmentation into dimers, completely absent for the trimer photodissociation [16], clearly indicates some internal conversion since electronic relaxation is required for the production of dimers. In a previous study [20], it was shown that thermodissociation (i.e., when all the photon energy has been transformed into vibrational excitations in the ground electronic state) leads dominantly to dimer fragments. Therefore the relative abundance of the monomer and dimer fragments is related to the efficiency of the internal conversion. Smaller fragments indicate less electronic relaxation and more violent photodissociation events. 


\section{Fragmentation patterns}

The results of our study are summarized in Figs. 5 and 6, where the relative abundances of the photofragments are reported as a function of the photon energy for various diatomics-inmolecules modelings and dynamical approaches. Previous theoretical [20] and experimental [21] results are also included for comparison. Fig. 5 represents MF dynamical calculations with diatomics-in-molecules modelings including the spin-orbit interactions (DIM+SO) or ignoring them (DIM), as used in most theoretical studies devoted to ionic argon clusters. While in Fig. 6, only one model is used (DIM+SO), various dynamical approaches are explored.

The global evolution of the photofragment abundances is rather intuitive. When the energy of the photon increases, the fragments produced are smaller as the energy deposited in the system becomes too high for large fragments to survive, and the smallest possible fragments, monomers, are produced. Only the ions are reported here since only the ions were measured; however, it is clear that the neutral atoms are loosely bound and obviously neutral clusters generally do not survive. Therefore the complementary fragments can always be considered the neutral monomers.

Surprisingly, for the MF dynamics and including the spin-orbit interaction, trimers are formed for photon energies around $3 \mathrm{eV}$; however, these trimers are electronically excited, metastable, and could decay radiatively or non-radiatively. Unfortunately, no experiments have been carried out at these photon energies to be compared with. We have also observed a similar situation for krypton and xenon clusters [32], where trimers with very long nonradiative life times were found in our simulations on the post-ionization fragmentation at energies for which the atomic fragments switch from the lower fine-structure state $\left({ }^{2} P_{3 / 2}\right)$ to the higher one $\left({ }^{2} P_{1 / 2}\right)$. Without the spin-orbit interactions included, these metastable trimers are almost not produced. At the lower photon energies, the ones of interest for a comparison with the experimental photodissociation data, no trimers are formed for any modeling and dynamical approach we have used. This is in a good agreement with the experiment where only monomers and dimers were observed as ionic photofragments.

Concerning the dimers produced, the spin-orbit coupling has only a little effect and ignoring it does not lead to large discrepancies. The spin-orbit coupling is small for argon and this can thus be expected (but, obviously is not the case for the heavier rare gases, krypton and 
xenon). A bit larger differences between the SO and no SO data are seen for the lower photon energies between $1.8 \mathrm{eV}$ and $2.3 \mathrm{eV}$. The agreement with the experimental data is generally very good for this lower experimental photon energy range and somewhat deteriorates above $2.4 \mathrm{eV}$ where we slightly underestimate the experimental dimers abundances.

There is a big difference with the previous calculation [20], although similar methods are employed for the modeling of intra-cluster interactions (DIM without SO), dynamics (MF), and also in the photoabsorption simulation (vertical excitations weighted by the absorption probability calculated from the transition dipole moment and Monte Carlo samplings at rather low temperatures). The previous authors related the disagreement between their theoretical and literature experimental data to an underestimation of the internal conversion within the MF (or HWD in Ref. 20) approach. We have checked that, in fact, this difference originates from the way the results are gathered. In the MF approach, fragments have a noninteger charge as illustrated in Fig. 7. This fractional charge varies between 0 (neutrals) and 1 (ions). Here, we have taken into account all the fragments weighted by their fractional charges, whereas in the previous theoretical study, only trajectories leading to fragments with charges not too far from 1 or 0 were accepted and the other trajectories were discarded from the statistics. We have checked here that, for $E_{\text {phot }}=2.15 \mathrm{eV}$, accepting the ion charge in the range of $0.8-1$ leads, within a few percent, to the same results as in Ref. 20, i.e., much less dimers and much more monomers. Therefore, the conclusion that MF leads to an underestimation of the internal conversion is not so clear. We have shown here that if all fractional charges are accepted in the statistics (and properly weighted), the MF results are fairly good for the relative abundances of photofragments. Obviously, these non-physical fractional charges forbid the evaluation of the internal energy of the fragments and also of their survival times since the potential energy surfaces of the neutrals and the ions are very different from each other and should not be mixed.

For ionic monomers, we have in general a fraction complementary to the ionic dimer abundance, except for the region of photon energies around $3 \mathrm{eV}$ where metastable trimers are produced if the spin-orbit coupling is considered at the expense of monomers (instead of dimers which are anyway almost absent for photon energies larger than $2.6 \mathrm{eV}$ ). In figure 5 and 6 , the statistics have been made after 50 ps. The trimers, as they are metastable, may decay into either dimers or monomers, which will be discussed in the next section.

In Fig. 6, we focus on the comparison with the experimental results for various dynamical 
approaches, all using the more realistic DIM+SO model. The MF dynamics data (grey line) are compared with the MFQ results calculated for two particular methods including quantum decoherence as introduced in Sec. II, namely MFQ-AMP/S and MFQ-TFS/G. The MFQAMP/S method is only slightly more time consuming than the MF approach, while the MFQ$\mathrm{TSH} / \mathrm{G}$ is much more time consuming since the non-adiabatic couplings and energy gradients should be evaluated. The MF and MFQ-AMP/S dynamical approaches lead to very similar results in the whole range of photon energies and the agreement with the experiment is good for the lower part of the photon energy window. The decrease of the dimers produced as the photon energy increases is nicely reproduced by the three methods qualitatively and almost quantitatively. The MFQ-TSH/G method leads to a better agreement with the experimental data in the high energy window, $E_{\text {phot }}>2.3 \mathrm{eV}$, but this agreement somewhat deteriorates for lower photon energies, for which the MFQ-TSH/G method slightly overestimates the abundance of dimer photofragments. The MF and MFQ-AMP/S, on the other hand, perform well below $2.3 \mathrm{eV}$ and underestimate the dimer abundance for $E_{\mathrm{phot}}=2.3-2.7 \mathrm{eV}$. At the higher experimental photon energies, if the metastable trimers all decayed in the dimer channel, the agreement with the experiment would be greatly improved for the MF and MFAMPS/S methods. Globally, the agreement with experimental relative abundances is quite good and satisfactory, and confirms that the internal conversion is very efficient at the lower photon energies and declines as the absorbed energy increases, which is demonstrated by the enhanced production of the smallest, monomer fragments. The global agreement is slightly better when the decoherence (or quenching) is driven by the non-adiabatic coupling (MFQTFS/G) rather than by the amplitudes of the various adiabatic states (MFQ-AMP/S). In addition, much less metastable trimers are produced with the MFQ-TSH/G method at high photon energies.

As emphasized previously, the MF approach leads to fragments with fractional charges. This is illustrated in Fig. 7, where the charge of the fragments is reported for three representative photon energies of the main visible band, 2.15, 2.35 and $2.65 \mathrm{eV}$. As can be seen, the charge is spread over almost all fractional values with a rather flat distribution for the MF method, while, for a MFQ method (MFQ-AMP/S), it is concentrated to either 0 (neutrals) or 1 (ions) with a very low residual background for the monomers. Only results for the MFQ-AMP/S are reported here, but the same holds for all the variants of the MFQ dynamical approach. 


\section{Kinetic energy distributions}

In the experiment, the kinetic energy distribution (KED) of the ionic fragments is measured and shows a clear bimodal structure for the monomers, similar to the photodissociation of $\mathrm{Ar}_{3}^{+}$for which slow and fast atoms can be identified, and a single peak for the dimers $[21,44]$. The results of our simulations at four selected photon energies $(2.15,2.35,2.55$, and $2.75 \mathrm{eV}$ ) are reported in Fig. 8. Except for the lowest photon energy, there are clearly two peaks in the KED of the monomers, either charged or neutral, one for zero kinetic energy and one for high kinetic energies, the latter shifting to higher values as the photon energy increases. We clearly get fast and slow monomers, as in the experimental KED. Again, the MFQ-AMP/S method gives results very similar to the MF approach, except for the lowest photon energy. Here, only the moduli of velocities have been taken into account not their directions. However, in the experiment, the fragments emitted with their velocity along the accelerating field of the time-of-flight apparatus will arrive first, while the ones with the velocity in the opposite direction are first decelerated and will arrive after the previous ones as well as after the ones in rest in the cluster center-of-mass. This will produce a central peak (slow fragments) and two wing peaks (fast fragments moving along and opposite to the accelerating field) in the KED record. This bimodal KED for monomer fragments evokes the trimer explosion which clearly imprints also the photodissociation of $\mathrm{Ar}_{5}^{+}$. However, we should have in mind that the atoms of the neutral crown are involved in the photoabsorption, and, therefore, the picture consists of a more distorted trimer, the explosion of which is accompanied by multiple collisions. Noteworthy, except for the lowest energy, the KED for neutral and ionic monomers are rather similar to each other showing that there have been large charge exchanges during the dissociation. Of course, the relative intensity of fast and slow fragments is different for ions and for neutral monomers since there is a single charged atom and four neutral monomers for each photodissociation event.

For the lowest photon energy, mainly $\mathrm{Ar}_{2}^{+}$fragments are produced, a smooth internal conversion efficiently occurs and the neutral monomers present a long tail in the KED evoking smooth evaporation. The MF approach leads to a large fractional charge contamination (see Fig. 7) and the KED is more affected. With the MFQ dynamical methods, these artifacts are removed. The ionic dimers produced have dominantly a low kinetic energy, in agreement with experimental findings. For example, measurements on the kinetic energy 
of the $\mathrm{Ar}_{2}^{+}$fragment and neutral atoms ejected from $\mathrm{Ar}_{5}^{+}$photoexcited at $532 \mathrm{~nm}$ (approx. $2.33 \mathrm{eV}$ ) yielded rather slow ionic dimers with kinetic energy release about $0.12 \mathrm{eV}$, although they could, in principle, acquire up to $1 \mathrm{eV}$ [44]. However, for the lowest photon energy, again, a rather long tail is obtained, confirming a rather smooth thermalization during the dissociation.

\section{Stability of ionic dimer and trimer fragments}

The analysis of the internal energy [45] of the ionic fragments is reported in Fig. 9 for dimers and in Fig. 10 for trimers. Only data obtained for the MFQ-AMP/S dynamical method are included in these figures since it is much less time consuming than the MFQ-TSH/G method and because such analysis is impossible for the MF approach due to the fractional charges. The rotational and vibrational internal energy of dimers displays a thermal-like distribution with rather cool underlying temperatures. As the energy of the initial photon increases, the distributions, both for rotations and vibrations, become colder, which indicates that the excess energy is more efficiently transformed into translational energy of the fragments, mainly monomers. As can be seen in Fig. 9c, the total internal energy of the ionic dimer fragments increases as the photon energy increases, mainly due to higher electronic states of the dimer involved. The total internal energy of the ionic dimer fragments has a broad distribution with a peak around the lower fine-structure dissociation limit at low photon energies and moving gradually to the higher fine-structure dissociation limit as the photon energy increases. However, only a very small proportion of the dimers have internal energies above these asymptotes and can be considered unstable, except for the highest photon energy for which dimers are produced in excited electronic states. The ionic trimers produced at high visible photon energies $(2.65 \mathrm{eV}$ and $2.85 \mathrm{eV})$ are very cool both rotationally and vibrationally $\left(E_{\text {rot }}<17 \mathrm{meV}\right.$ and $E_{\text {vib }}<53 \mathrm{meV}$ with mean values $\bar{E}_{\text {rot }} \approx 2 \mathrm{meV}$ and $\bar{E}_{\text {vib }} \approx 14 \mathrm{meV}$ for both photon energies), therefore only their total internal energy is displayed in Fig. 10. All are metastable with respect to the dissociation into the electronic ground-state ionic dimer and their internal energy is even above the lowest atomization energy corresponding to the lowest fine-structure atomic state of the ionic monomer.

In principle, an unstable or metastable ionic trimer can eventually dissociate either into 
an ionic dimer and a neutral monomer, or into three monomers, one ionic and two neutral [46]. Four dissociative processes,

$$
\begin{gathered}
\operatorname{Ar}_{3}^{+} \rightarrow \operatorname{Ar}_{2}^{+}\left(I(1 / 2)_{\mathrm{u}}\right)+\mathrm{Ar}, \\
\mathrm{Ar}_{3}^{+} \rightarrow \operatorname{Ar}_{2}^{+}\left(I I(1 / 2)_{\mathrm{u}}\right)+\mathrm{Ar}, \\
\mathrm{Ar}_{3}^{+} \rightarrow \mathrm{Ar}^{+}\left({ }^{2} P_{3 / 2}\right)+\mathrm{Ar}+\mathrm{Ar} \\
\mathrm{Ar}_{3}^{+} \rightarrow \operatorname{Ar}^{+}\left({ }^{2} P_{1 / 2}\right)+\mathrm{Ar}+\mathrm{Ar}
\end{gathered}
$$

are representative for the decay of ionic trimers. As discussed already in an earlier study [15], the electronic states of the rare-gas clusters cations available within the diatomics-inmolecules modeling split up into two groups if the spin-orbit coupling is included: a group of states asymptotically correlating to ${ }^{2} P_{3 / 2}$ atomic ions (in general, lower $2 / 3$ of the electronic states, and specifically for $\mathrm{Ar}_{3}^{+}$, the electronic ground state and five lowest excited states) and another group correlating asymptotically to ${ }^{2} P_{1 / 2}$ atomic ions (remaining $1 / 3$ of the electronic states, i.e., the highest three excited states available for $\mathrm{Ar}_{3}^{+}$within the DIM+SO model). While the two groups interact only weakly with each other, non-negligible nonadiabatic couplings are observed between the states belonging to each particular group. Consequently, the dynamics of an $\mathrm{Rg}_{N}^{+}$cluster is more or less quasi-adiabatic with respect to switches between the two groups of states [14, 24]. This quasi-adiabaticity is the more pronounced the stronger is the spin-orbit coupling, i.e., it is rather strictly followed in heavier rare gases, krypton and xenon, and much weaker, but still well apparent in argon. Taking all this into account, process 4 represents the energetically most favorable way for the fragmentation of electronically excited trimer cations occupying one of the six states belonging to the ${ }^{2} P_{3 / 2}$ group, process 5 plays the same role for trimers populating one of the three excited states of the ${ }^{2} P_{1 / 2}$ family, and processes 6 and 7 represent two possible paths for the complete atomization of the ionic trimer. Minimal internal energies required for the dissociation of the ionic trimer via processes $5-7$ are included in Fig. 10 as vertical lines, the energy required for process 4, completely outside the range of internal energies included in Fig. 10, is given in the figure caption. Clearly, all the ionic trimers produced from $\mathrm{Ar}_{5}^{+}$clusters excited at the excitation energies considered in Fig. 10 can dissociate via the energetically most favorable channel 4, and are thus not stable. An absolute majority of them can even fragment via the monomer channel 6, and a smaller sub-population of 
the ionic trimer fragments (right-hand-side peaks in Fig. 10) has enough energy to produce highly electronically excited dimers via channel 5. Apparently, channel 7 is energetically closed for the two energies considered in Fig. 10).

To investigate the stability of the ionic trimers produced from $\mathrm{Ar}_{5}^{+}$clusters excited at selected photon energies between $E_{\text {phot }} \approx 2.65-3.15 \mathrm{eV}$ more thoroughly, we have extended the propagation of the respective trajectories, i.e., those ending in the ionic trimer at $t=$ $50 \mathrm{ps}$, up to $t=200 \mathrm{ps}$. The results we have obtained are summarized in Table II where relative abundances of the ionic trimer fragments detected at $t=50 \mathrm{ps}$ and $t=200 \mathrm{ps}$ are compared to each other for both MFQ methods employed. In addition, the fractions of the trimers produced at $t=50 \mathrm{ps}$ which dissociate during the extended time evolution are provided as values in parentheses. A basic conclusion that can be drawn from Table II is that not all trimers, even though having enough energy, dissociate within the extended time window. Moreover, even after the time propagation has been extended for the MFQ$\mathrm{AMP} / \mathrm{S}$ method and for $E_{\text {phot }}=2.85 \mathrm{eV}$ over further $200 \mathrm{ps}$ (up to $t=400 \mathrm{ps}$ ), a substantial portion of the ionic trimer fragments has survived (only $31 \%$ of the ionic trimers detected at $t=50 \mathrm{ps}$ dissociate by $t=400 \mathrm{ps}$ ). After fitting the relative abundances of the ionic trimers we have obtained for $E_{\text {phot }}=2.85 \mathrm{eV}$ from this long, $400 \mathrm{ps}$ calculation to an exponential decay function, we get for the asymptotic abundance of $\operatorname{Ar}_{3}^{+} f(t \rightarrow \infty)=0.234 \pm 0.004$ (i.e., a $34 \%$ decay), and the exponential decay time $\tau=(116 \pm 10)$ ps. If the same is performed for the MFQ-TFS/G method and $E_{\text {phot }}=2.85 \mathrm{eV}$ (but only for times $t \leq 200 \mathrm{ps}$ included because of higher computational demands for the MFQ-TFS/G method), we get $f(t \rightarrow \infty)=$ $0.076 \pm 0.007$ (a $47 \%$ decay with respect to $t=50 \mathrm{ps}$ ) and $\tau=(77 \pm 19)$ ps. Consequently, a non-negligible amount of the ionic trimer fragments survive even for long integration times, much less for the MFQ-TFS/G method than for MFQ-AMP/S, and according to calculated exponential decay times, the dissociation dynamics of $\mathrm{Ar}_{3}^{+}$should be saturated within several hundreds of pico-seconds.

But, what about the trimer fragments which survive for rather long times even though carrying enough energy to disintegrate to three separate monomers? As discussed above, the argon trimer can populate two only weakly interacting groups of electronic states. It follows from our calculations, that the trimers produced from $\mathrm{Ar}_{5}^{+}$excited by photons at energies between $E_{\text {phot }} \approx 2.65-3.15 \mathrm{eV}$ occupy almost exclusively the higher group of states and can, thus, efficiently fragment only via paths 5 and 7 . The latter is energetically 
closed, however, for the excitation energies considered (see Fig. 10) and only a smaller part of the ionic trimers have enough energy to go through path 5 (right-hand peaks in Fig. 10). The remaining trimer fragments can efficiently dissociate only after switching from the upper group of electronic states to the lower group. But, due to a very weak non-adiabatic coupling between the electronic states belonging to the upper group with the states of the lower group, this would take an extremely long time compared to the integration times accessible in our calculations. In the experiment, on the other hand, such long times can be reached and, consequently, experimental trimer fragments could comfortably dissociate before being analyzed. An alternative way of disintegrating the electronically metastable trimers is the radiative decay. Leaning upon recent experimental findings for ionized argon and neon dimers [47], and ionic trimers of krypton [48], we estimate characteristic times for the radiative decay of metastable $\mathrm{Ar}_{3}^{+}$to dozens of micro-seconds. Again, times which are accessible in a typical experimental setup. Noteworthy, as there are no separate groups of electronic states for the DIM model (i.e, with the spin-orbit coupling not included), almost no ionic trimers are produced in this case (see Fig. 5).

In the end of the stability analysis on the ionic trimer fragments, let us briefly discuss why they are emitted from excited $\mathrm{Ar}_{5}^{+}$at the excitation energies ranging between $2.65-3.15 \mathrm{eV}$. The situation is quite similar to what we observed recently for the photodynamics of rare-gas trimer cations [14]. As is well known, ionic rare-gas trimers dissociate, upon photoexcitation, via a monomer channel, either channel 6 for lower photon energies or channel 7 for higher VIS and UV photon energies. However, in our former calculations [14], we detected ionic dimers among the photofragments for a narrow interval of photon energies around $2.5 \mathrm{eV}$. The reason was simple. Exactly at these photon energies, the upper family of the electronic states of $\mathrm{Rg}_{3}^{+}$starts to be populated after photoexcitation and, as the energy pumped to the ionic trimer may not be sufficient for monomer channel 7 in this energy range, the disintegrating trimer prefers energetically more favorable path 5 . For higher photon energies, the monomer channel 7 opens, however, and the trimer photodissociation switches to this paths. Noteworthy, the onset of the production of ionic trimers from photoexcited $\mathrm{Ar}_{5}^{+}$at $E_{\text {phot }} \approx 2.6-2.7 \mathrm{eV}$ coincides well with the energy of the lowest electronic state belonging to the upper group of electronic states of $\mathrm{Ar}_{5}^{+}$(state 11 of Table I). The argon pentamer prefers, for the photon energies close to this lower limit, the more energetically favorable dissociation path via an ionic trimer for exactly the same reasons as the ionic trimer selects 
the dimer channel at $E_{\text {phot }} \approx 2.5 \mathrm{eV}$.

Most of the ionic dimer fragments released from photoexcited $\mathrm{Ar}_{5}^{+}$are stable for lower photon energies and, similarly to what we observe for trimers, unstable for the photon energies above $\approx 2.65 \mathrm{eV}$. Indeed, if we inspect Fig. 9, we clearly see that only minor part of the ionic dimers carry enough energy to dissociate to $\operatorname{Ar}^{+}\left({ }^{2} P_{3 / 2}\right)$ and no electronically excited $\mathrm{Ar}^{+}\left({ }^{2} P_{1 / 2}\right)$ can be produced for lower photon energies, while, for higher photon energies, $E_{\text {phot }} \geq 2.65 \mathrm{eV}$, the production of electronic ground-state $\operatorname{Ar}^{+}\left({ }^{2} P_{3 / 2}\right)$ ions opens, but still only a minority of ionic dimer fragments have enough energy to emit electronically excited $\operatorname{Ar}^{+}\left({ }^{2} P_{1 / 2}\right)$. This conclusion is further confirmed by the data collected in Table III, where relative fractions of unstable and/or metastable ionic dimer fragments detected at the end of $50 \mathrm{ps}$ trajectories , $\Delta f_{\mathrm{r}}$, are given for selected photon energies. To see the evolution of the dimer fragments which have enough energy to further dissociate, we extended the integration of the trajectories resulting at $t=50 \mathrm{ps}$ in an ionic dimer and three neutral monomer fragments up to $t=200$ ps. Note, however, that the total abundance of $\mathrm{Ar}_{2}^{+}$ can change in two independent ways: a) it decreases due to the unstable dimers decay or b) it can increase due to the production of dimers from unstable trimers. Interestingly, for both dynamical methods, the total abundances of ionic dimers increase with time because of prevalence of the trimer decay channel over the ionic dimer decay. In Table III, we provide both the total change of the ionic dimer fragments, $c f$. columns $f(50 \mathrm{ps})$ and $f(200 \mathrm{ps})$ of Table III, as well as relative changes of the ionic dimer fragments abundance via the dimer and trimer decay (values given in parentheses). An interesting observation can be made considering the data of Table III. Namely, for the two lowest photon energies, $E_{\text {phot }}=2.15 \mathrm{eV}$ and $E_{\text {phot }}=2.35 \mathrm{eV}$, only a marginal number of unstable ionic dimers is produced and, in addition, almost all of them disintegrate within the extended simulation time. These dimers are thus dynamically unstable. For the border-line energy, $E_{\text {phot }}=2.65 \mathrm{eV}$, and, in particular, for the two higher energies, $E_{\text {phot }}=2.85 \mathrm{eV}$ and $E_{\text {phot }}=2.95 \mathrm{eV}$, many ionic dimers carrying enough energy to further dissociate are produced, on the other hand, but only a small portion of them disintegrates if the calculation is prolonged up to $t=200 \mathrm{ps}$ (or even extrapolated to $t \rightarrow+\infty)$. Consequently, only a minor part of the unstable ionic dimers produced at higher photon energies are virtually dynamically unstable and most of them are metastable and represent long lived fragment species. The explanation for this observation is similar to what we have proposed for the trimer fragments. At photon energies above 
$E_{\text {phot }} \approx 2.65 \mathrm{eV}$, the upper family of electronic states of the ionic dimer fragments starts to be populated and only a few of them carry enough energy to dissociate to $\operatorname{Ar}^{+}\left({ }^{2} P_{1 / 2}\right)$ monomers (exactly those dimers fragmenting in our calculations after the time evolution is extended to $t=200 \mathrm{ps}$ ). The remaining dimer fragments can only dissociate to energetically less rich $\mathrm{Ar}^{+}\left({ }^{2} P_{3 / 2}\right)$ monomers, but due to the strong adiabatic separation of the dimer states correlating asymptotically to $\operatorname{Ar}^{+}\left({ }^{2} P_{3 / 2}\right)$ from the states correlating to $\operatorname{Ar}^{+}\left({ }^{2} P_{1 / 2}\right)$, such a decay would require much longer times than are accessible in any trajectory calculation. Or, alternatively, a switch from the upper family to the lower family of electronic states must occur prior to subsequent dimer explosion via a radiative transition. According to recent estimates [47], such a radiative transition would require dozens of microseconds.

\section{Photodynamics in the UV absorption band}

In addition to the VIS absorption band, the $\mathrm{Ar}_{5}^{+}$cluster absorbs also, though much less efficiently, in the UV part of the electromagnetic spectrum, approximately around $4.5 \mathrm{eV}$ of photon energy. As is clear from panel (b) of Fig. 4, only one electronic transition is responsible for this UV absorption, namely the transition from the electronic ground state to $14^{\text {th }}$ excited state of $\mathrm{Ar}_{5}^{+}$(the highest excited state available within the diatomics-inmolecules models used in this work). Noteworthy, the positive charge delocalization in this highly excited state is the same as in the electronic ground state of $\mathrm{Ar}_{5}^{+}$for both structural isomers of $\mathrm{Ar}_{5}^{+}$relevant at the temperature considered here, isomer 1 and isomer 2 of Tab. I. The charge resides on the three atoms belonging to the ground-state ionic core and the two outer atoms remain almost neutral even after excitation. Consequently, only the ionic core of the cluster is involved in the photoabsorption and the same can be expected for the ensuing photodissociation. More specifically, the three charged atoms undergo, after the excitation, a trimer-like explosion while the two neutral atoms play a role of mere spectators. Of course, a charge transfer can occur and make the picture a bit more involved, but we do not expect this to be of a remarkable importance as the fragmentation processes will be extremely fast at the UV photon energies. A direct conclusion of the preceding analysis is that the UV photofragmentation of $\mathrm{Ar}_{5}^{+}$should be rather similar to what is observed for the argon trimer cation, $\mathrm{Ar}_{3}^{+}[13,49]$. Asymmetric stretching of the ionic core, leading to $\mathrm{Ar}_{2}^{+} \ldots$ Ar configurations, is highly probably responsible for the photoabsorption of $\mathrm{Ar}_{5}^{+}$in 
the UV region, alike for $\mathrm{Ar}_{3}^{+}$, and results to the dissociation of the core into three monomer fragments. For asymmetric ionic cores, both fast and slow ionic monomer fragments will be produced as well as fast and slow neutral monomers. The two neutral spectator atoms of $\mathrm{Ar}_{5}^{+}$are expected to contribute to the slow neutral monomer fragments signal.

These qualitative conclusions are confirmed by our simulation data. The data of Table IV clearly show that the ionic monomer fragments dominate for UV photon energies (about 95\% of ionic fragments). The small portion of the ionic dimer fragments (about 5\%) must be due to the charge transfer to the two originally neutral spectator atoms. Note also that the abundance of the ionic dimers is higher for the MFQ models which can be expected to lead to a more efficient charge transfer due to the quenches of the electronic wavefunction. Interestingly, the fact that the excited states which interact strongly with the highest excited state, states 11 - 14 of Table I, have a substantial portion of the positive charge deposited on the spectator atoms complies well with this expectation. In addition, the ionic dimer fragments observed at the end of the 50 ps trajectories in the UV region are all metastable. They are electronically excited but trapped in minima on the excited potential energy surfaces of $\mathrm{Ar}_{2}^{+}$. They have enough energy to dissociate into monomers, but only after hopping from the upper family of the $\mathrm{Ar}_{2}^{+}$electronic states (states II [28]) to the lower family of states (states I [28]). As the two families of states are rather weakly coupled, such hops are highly improbable if only radiationless transitions are allowed (as is the case of the present calculation). Radiative processes are thus expected, like for trimers, to be responsible for the subsequent decay of the ionic dimer photofragments.

Calculated distributions of fragments kinetic energies are in an excellent agreement with our qualitative predictions, see Fig. 11. Fast as well as slow monomer fragments, both ionic and neutral, are produced in the UV region, which closely resembles the patterns observed for the $\mathrm{Ar}_{3}^{+} \mathrm{UV}$ photofragmentation $[14,16]$. Somewhat surprisingly, no fine structure of the fast fragments peak is observed for $\mathrm{Ar}_{5}^{+}$, unlike for trimer (see Fig. 2 of Ref. 14). This observation seems to indicate that either internal collisions in the ionic core of photoexcited $\mathrm{Ar}_{5}^{+}$are not as important during early stages of the fragmentation process as in $\mathrm{Ar}_{3}^{+}$or, more probably, that involved collision dynamics emerges in this case which completely removes the fine structure of the high-energy peak of the kinetic energy distribution. Only slow ionic dimer fragments are detected, on the other hand, in the UV region of photon energies, which can also be easily understood as the ionic dimers are expected to be formed with the 
participation of the two spectator atom aside the ionic core via the charge transfer.

Ionic trimers are produced from the UV excited $\mathrm{Ar}_{5}^{+}$clusters only at a marginal amount and, consequently, need not be considered. Moreover, they are all dynamically unstable and will eventually dissociate if the trajectories passing through the trimer channel at $t=50 \mathrm{ps}$ are extended to larger times.

\section{CONCLUSIONS}

We have performed a complete theoretical investigation of the photodissociation dynamics of $\mathrm{Ar}_{5}^{+}$using previously developed methodologies for modeling the intra-cluster interactions and recent dynamical approaches. The thermodynamics and absorption properties of the cluster have been investigated. Although various structural isomers have been found for $\mathrm{Ar}_{5}^{+}$, only the ones with a linear trimer core are expected to play a significant role under realistic conditions. This trimer core represents the chromophore for the photon absorption, the remaining atoms also participate in the absorption, however, due to charge transfer in electronically excited states, particularly in the main visible band. The photodissociation dynamics is more involved than for the trimer cluster and, here, rapid and slow processes compete such as internal conversion, fission of the chromophoric core, and evaporation of neutral atoms.

At lower visible photon energies, internal conversion is the most effective process and leads dominantly to ionic dimer fragments, while at higher visible photon energies, the efficiency of the internal conversion attenuates and ionic monomer fragments prevail. The photodynamics is imprinted by the explosion of the chromophoric trimer core which leads to fast monomer fragments; however, the other atoms also participate, instead of remaining mere spectators, and large charge migrations occur. At the higher visible photon energies, the spin-orbit coupling should be considered. More specifically, metastable ionic trimers are produced at the expense of ionic monomers at the onset of the production of fine-structure excited atomic fragments if the spin-orbit coupling is considered.

Noteworthy, the mean-field approach shows a satisfactory behavior with the photon energy for the internal conversion efficiency, almost in quantitative agreement with the detailed experimental data for the monomer/dimer abundances, but only if all fragments are considered in the statistics, even those with fractional charges far from the physical values, either 
0 (neutrals) or 1 (ions). The new dynamical methods involving periodic decoherence nicely solve the epinous problem of the fractionally charged fragments and are also in satisfactory agreement with the experimental data. Moreover, the new methods allow for an analysis of the internal energy and stability of photofragments. For example, the non-radiative lifetime of the metastable trimers has been estimated to be about $100 \mathrm{ps}$.

For the UV excitation, the photodissociation is more similar to the trimer case. It is dominated by the fast explosion of the ionic core, however, no trimodal (pentamodal for the time-of-flight spectra) kinetic energy distribution is found here for the ionic monomer fragments. Instead, simpler bimodal distributions (trimodal for the TOF) have been obtained from our calculations in this spectral region.

This study provides very encouraging results and strong grounds for all the methodological approaches involved, from the modeling of the cluster potential energy surfaces and non-adiabatic couplings via the diatomics-in-molecules approach to semiclassical absorption simulation and the multi-surfaces and multi-degrees of freedom dynamics using the meanfield approach and periodic decoherence. It would be very interesting if similar detailed results with extended photon energy range could be available for larger ionic argon clusters and also for heavier rare gases for which the spin-orbit interactions are expected to play a larger role.

\section{ACKNOWLEDGMENTS}

The work was financially supported by the Grant Agency of the Academy of Sciences of the Czech Republic (grant no. IAA401870702), the BARRANDE program (grant no. 7329/2009-32), and the University of Ostrava (grant no. SGS7/PřF/2010). The calculations were carried out on the computers of the Supercomputing Center of the Technical University of Ostrava and on the computers of the Center of Numerically Demanding Calculations of the University of Ostrava, financially supported by the Ministry of Education, Youth and Sports of the Czech Republic (grant No. 1N04125). Last but not least, our thanks belong to D. Hrivňák, who programmed substantial parts of the code we used, and to I. Janeček, 
University of Ostrava, who improved the implementation of the MFQ-TFS/G method.

[1] H. Haberland, ed., Clusters of Atoms and Molecules, vol. 52 of Springer. Ser. Chem. Phys. (Springer, Berlin, 1994).

[2] P. J. Kuntz and J. Valldorf, Z. Phys. D 8, 195 (1988).

[3] M. Amarouche, G. Durand, and J. P. Malrieu, J. Chem. Phys. 88, 1010 (1988).

[4] T. Ikegami, T. Kondow, and S. Iwata, J. Chem. Phys. 93, 3038 (1993).

[5] F. Y. Naumkin, P. J. Knowles, and J. N. Murrell, Chem. Phys. 193, 27 (1995).

[6] J. Galindez, F. Calvo, P. Paška, D. Hrivňák, R. Kalus, and F. X. Gadea, Comput. Phys. Commun. 145, 126 (2002).

[7] A. B. Jones, A. L. M. Buxey, P. R. Jukes, J. A. Smith, and A. J. Stace, J. Chem. Phys. 103, $474(1995)$.

[8] V. Lepère, Y. J. Picard, M. Baraf, J. A. Fayeton, B. Lucas, and K. Béroff, J. Chem. Phys. 130, Art. No. 194301 (2009).

[9] F. O. Ellison, J. Am. Chem. Soc. 85, 3540 (1963).

[10] M. Amarouche, F. X. Gadea, and J. Durup, Chem. Phys. 130, 145 (1989).

[11] M. Amarouche and F. X. Gadea, Chem. Phys. 140, 385 (1990).

[12] F. X. Gadea, Z. Phys. D 20, 25 (1991).

[13] A. Bastida and F. X. Gadea, Z. Phys. D 39, 325 (1997).

[14] D. Hrivňák, R. Kalus, and F. X. Gadea, Phys. Rev. A 79, Art. No. 013424 (2009).

[15] D. Hrivňák, R. Kalus, and F. X. Gadea, Europhys. Lett. 71, 42 (2005).

[16] H. Haberland, B. von Issendorf, and A. Hofmann, J. Chem. Phys. 103, 3450 (1995).

[17] F. X. Gadea and M. Amarouche, J. de Phys. II France 5, 1767 (1995).

[18] A. Bastida and F. X. Gadea, Chem. Phys. 209, 291 (1996).

[19] F. Calvo, J. Galindez, and F. X. Gade, J. Phys. Chem. A 106, 4145 (2002).

[20] F. Calvo, J. Galindez, and F. X. Gadea, PCCP 5, 321 (2003).

[21] T. Nagata, J. Hirokawa, and T. Kondow, Chem. Phys. Lett. 176, 526 (1991).

[22] P. Ehrenfest, Z. Phys. 45, 455 (1927).

[23] J. C. Tully, J. Chem. Phys. 93, 1061 (1990).

[24] I. Janeček, S. Cintavá, D. Hrivňák, R. Kalus, M. Fárník, and F. X. Gadea, J. Chem. Phys. 
131, Art. No. 114306 (2009).

[25] J. S. Cohen and B. I. Schneider, J. Chem. Phys. 61, 3230 (1974).

[26] N. Doltsinis and P. Knowles, Mol. Phys. 94, 981 (1998).

[27] To illustrate this fact more clearly, we use the DIM+SO model with the polarization threebody forces (the induced dipole - induced dipole interaction of Ref. [3]) included in spectroscopic calculations of Sec. III A. Therein, we denote this, in such a way extended interaction model as DIM+SO+ID-ID.

[28] F. X. Gadea and I. Paidarová, Chem. Phys. 209, 281 (1996).

[29] R. A. Aziz, J. Chem. Phys. 99, 4518 (1993).

[30] C. Yamada, H. Kanamori, and E. Hiroka, J. Chem. Phys. 83, 552 (1985).

[31] D. Hrivňák and R. Kalus, Chem. Phys. 264, 319 (2001).

[32] I. Janeček, D. Hrivňák, R. Kalus, and F. X. Gadea, J. Chem. Phys. 125, Art. No. 104315 (2006).

[33] N. C. Blais and D. G. Truhlar, J.Chem Phys 79, 1334 (1983).

[34] T. Ikegami, T. Kondow, and S. Iwata, J. Chem. Phys. 98, 3038 (1993).

[35] F. Y. Naumkin, Chem. Phys. 252, 301 (2000).

[36] R. Kalus, I. Paidarová, D. Hrivňák, and F. X. Gadea, Chem. Phys. 298, 155 (2004).

[37] J. A. Gascón, R. W. Hall, C. Ludewigt, and H. Haberland, J. Chem. Phys. 117, 8391 (2002).

[38] For the pentameric geometry of Table 1, $\Delta Q$ also becomes equal to zero. But this high-energy structure need not be considered here. Not only that it will missing at lower temperatures considered in Fig. 2 due to high energy, but also it will be extremely rare at higher temperatures due to an only small part of the configuration space belonging to the basin of attraction of this structure.

[39] R. Kalus and D. Hrivňák, Chem. Phys. 278, 21 (2002).

[40] N. E. Levinger, D. Ray, K. K. Murray, A. S. Mullin, C. P. Schulz, and W. C. Lineberger, J. Chem. Phys. 91, 4019 (1989).

[41] It should be noted, however, that the temperature assessed from classical Monte Carlo calculations is highly probably overestimated due to the neglect of the quantum behavior of relatively light argon atoms, as discussed, e.g., in Ref. 6. The elevated "classical" temperature supplies then the quantum delocalization of argon nuclei missing naturally in classical calculations.

[42] J. T. Snodgrass, C. M. Roehl, and M. T. Bowers, Chem. Phys. Lett. 159, 10 (1989). 
[43] T. Nagata, J. Hirokawa, T. Ikegami, and S. Iwata, Chem. Phys. Lett. 171, 433 (1990).

[44] J. A. Smith, N. G. Gotts, J. F. Winkel, R. Hallet, C. A. Woodward, A. J. Stace, and B. J. Whitaker, J. Chem. Phys. 97, 397 (1992).

[45] Standardly, the internal energy is the sum of the electronic (interaction) energy and the kinetic energy of nuclei in the cluster center-of-mass frame. In this work, the zero of the interaction energy is identified with the energy of the fully dissociate state, $\mathrm{Ar}^{+}+N \mathrm{Ar}$, calculated for the spin-orbit coupling not considered.

[46] The latter channel may, of course, end also in an ionic monomer and a neutral dimer instead of two separate neutral monomers. Below we do not distinguish between these two variants, however, because the neutral argon dimer is only loosely bound by weak dispersion forces, as already discussed above for the neutral complexes in general.

[47] J. Fedor, R. Parajuli, S. Matt-Leubner, O. Echt, F. Hagelberg, K. Głuch, A. Stamatovic, M. Probst, P. Scheier, and T. D. Märk, Phys. Rev. Lett. 91, Art. No. 133401 (2003).

[48] J. Fedor, private communication.

[49] M. Deluca and M. A. Johnson, Chem. Phys. Lett. 162, 445 (1989). 


\section{Figures and Tables}

Fig. 1 Relative energies (ZPE included) and geometric and electronic structures of the stable and metastable isomers of $\mathrm{Ar}_{5}^{+}$as obtained for the cluster electronic ground state using the DIM+SO model. Darkness of individual atoms corresponds to the charge they carry (dark balls represent charged atoms belonging to the cluster ionic core, grey balls are used for almost neutral atoms). Energies of metastable isomers (2-5) relative to the stable isomer (1) are given in meV, charges are expressed in per cent of the elementary charge. Charges given in parentheses correspond to atoms aside the linear core of the cluster. Note that the dissociation energy for $\mathrm{Ar}_{5}^{+} \rightarrow \mathrm{Ar}_{4}^{+}+\mathrm{Ar}$ is equal to $+101 \mathrm{meV}$ for the $\mathrm{DIM}+\mathrm{SO}$ model.

Fig. 2 Temperature dependence of selected thermodynamical and structural parameters of $\mathrm{Ar}_{5}^{+}$as obtained from canonical MC simulations: (a) the heat capacity (with the constant kinetic energy contribution subtracted), (b) average distance of atoms from cluster center-of-mass, and (c) charge coefficient (see text for more details). In panel (b), full points correspond to the global average distance calculated for the cluster as a whole, open circles represent data calculated separately for the cluster ionic core; hatched or crosshatched areas correspond to temperatures for which monomer evaporation has been observed for $0.1 \%$ or $0.5 \%$ of generated MC samples, respectively.

Fig. 3 Relative abundances of the structural isomers of electronically ground-state $\mathrm{Ar}_{5}^{+}$clusters (see Fig. 1) calculated for thermally equilibrium cluster populations at various temperatures (panel a), and obtained for $T=50 \mathrm{~K}$ sub-populations selected for the photoexcitation at particular photon energies (panel b). The symbols used are as follows: circles - isomer 1, squares - isomer 2, triangles - isomer 3. Horizontal lines in panel (b) represent purely thermal abundances of isomers 1 and 2 at $T=50 \mathrm{~K}$.

Fig. 4 Photoabsorption spectrum of $\mathrm{Ar}_{5}^{+}$as calculated with the DIM+SO model. (a) Photoabsorption profiles obtained for various temperatures: $T=50 \mathrm{~K}$ - full line, $T=75 \mathrm{~K}$ - dashed line, $T=125 \mathrm{~K}$ - dash-dot line, and $T=175 \mathrm{~K}$ - dotted line. In the inset, calculations performed at $T=50 \mathrm{~K}$ for various interaction models are compared to each other: DIM - dotted line, DIM+SO - full line, and DIM+SO+ID-ID - dashed line. 
(b) Contributions of different electronically excited states to the overall photoabsorption spectrum of $\mathrm{Ar}_{5}^{+}$obtained for $T=50 \mathrm{~K}$ (grey line): dashed lines - states 2 and 3, full lines - states 4-12, dotted lines - states 13 and 14, and dot-dashed line - state 15 (see also Tab. I).

Fig. 5 Relative abundances of ionic photofragments produced in the main (VIS) absorption band of $\mathrm{Ar}_{5}^{+}$from $T=50 \mathrm{~K}$ clusters as obtained from MF calculations: $\mathrm{DIM}$ - up triangles, DIM+SO - down triangles. For comparison, depicted are also experimental values of Ref. 21 (full circles) as well as results of a previous MF study [20] (crosses).

Fig. 6 Relative abundances of ionic photofragments produced in the main (VIS) absorption band of $\mathrm{Ar}_{5}^{+}$from $T=50 \mathrm{~K}$ clusters as obtained for the DIM+SO model from MFQ calculations: MFQ-AMP/S - squares, and MFQ-TFS/G - circles. For comparison, experimental values of Ref. 21 (solid dots) and data obtained from MF calculations (grey line) are also shown.

Fig. 7 Normalized distributions of photofragments charges as obtained from MF calculations performed for the DIM+SO model, $T=50 \mathrm{~K}$, and three representative photon energies of the main absorption band: $2.15 \mathrm{eV}$ - empty bars, $2.35 \mathrm{eV}$ - hatched bars, and $2.65 \mathrm{eV}$ - crosshatched bars. In the insets, charge distributions are depicted for the MFQ-AMP/S dynamical method for comparison. The distributions are normalized so that for each photon energy they integrate up to relative abundances of the fragments.

Fig. 8 Normalized distributions of kinetic energies of ionic monomer fragments $\left(\mathrm{Ar}^{+}\right)$, neutral monomer fragments $(\mathrm{Ar})$, and ionic dimer fragments $\left(\mathrm{Ar}_{2}^{+}\right)$emitted from $\mathrm{Ar}_{5}^{+}$ photoexcited in the main (VIS) absorption band as calculated for $T=50 \mathrm{~K}$ using the MFQ-AMP/S dynamical method and the DIM+SO interaction model: $E_{\text {phot }}=2.15 \mathrm{eV}-$ solid line, $E_{\text {phot }}=2.35 \mathrm{eV}-$ dashed line, $E_{\text {phot }}=2.55 \mathrm{eV}-$ dotted line, and $E_{\text {phot }}=2.75 \mathrm{eV}$ - dash-dotted line. For comparison, distributions obtained from MF calculations are also added as grey lines. The distributions are normalized so that they integrate up for each photon energy to relative abundances of the fragments.

Fig. 9 Normalized distributions of (a) rotation, (b) vibration, and (c) total internal 
energy of ionic dimer fragments, $\mathrm{Ar}_{2}^{+}$, emitted from $\mathrm{Ar}_{5}^{+}$photoexcited in the main (VIS) absorption band as calculated for $T=50 \mathrm{~K}$ using the MFQ-AMP/S dynamical method and the DIM+SO interaction model: $E_{\text {phot }}=2.15 \mathrm{eV}-$ solid line, $E_{\text {phot }}=2.35 \mathrm{eV}-$ dashed line, $E_{\text {phot }}=2.55 \mathrm{eV}$ - dotted line, and $E_{\text {phot }}=2.75 \mathrm{eV}$ - dash-dotted line. Vertical grey lines in the $E_{\text {int }}$ panel correspond to $\operatorname{Ar}^{+}\left({ }^{2} P_{3 / 2}\right)+\operatorname{Ar}$ (full line) and $\operatorname{Ar}^{+}\left({ }^{2} P_{1 / 2}\right)+\operatorname{Ar}$ (dashed line) dissociation limits. The distributions have been obtained from 500 trajectories for $E=2.15 \mathrm{eV}$ and from 1,000 trajectories for the other photon energies (to improve convergence) and are normalized so that they integrate up for each photon energy to relative abundances of the fragments.

Fig. 10 Normalized distributions of total internal energy $\left(E_{\text {int }}\right)$ of ionic trimer fragments, $\mathrm{Ar}_{3}^{+}$, emitted from $\mathrm{Ar}_{5}^{+}$photoexcited in the main (VIS) absorption band as calculated for $T=50 \mathrm{~K}$ using the MFQ-AMP/S dynamical method and the DIM+SO interaction model: $E_{\text {phot }}=2.65 \mathrm{eV}$ - solid line and $E_{\text {phot }}=2.85 \mathrm{eV}$ - dashed line. Vertical grey lines correspond to $\operatorname{Ar}^{+}\left({ }^{2} P_{3 / 2}\right)+2 \operatorname{Ar}$ (full line) $\operatorname{Ar}^{+}\left({ }^{2} P_{1 / 2}\right)+2 \operatorname{Ar}$ (dashed line), and $\operatorname{Ar}_{2}^{+}(I I(1 / 2) u)+\operatorname{Ar}$ (dotted line) dissociation limits; note that all trimer fragments have internal energy much higher than the ground state of ionic dimer, $\operatorname{Ar}_{2}^{+}(I(1 / 2) u)$ $D_{\mathrm{e}}=-1.394 \mathrm{eV}$. The distributions have been obtained from 1,000 trajectories and are normalized so that they integrate up for each photon energy to relative abundances of the fragments.

Fig. 11 Normalized distributions of kinetic energies of ionic monomer fragments $\left(\mathrm{Ar}^{+}\right)$, neutral monomer fragments $(\mathrm{Ar})$, and ionic dimer fragments $\left(\mathrm{Ar}_{2}^{+}\right)$emitted from $\mathrm{Ar}_{5}^{+}$photoexcited in the UV absorption band as calculated for $T=50 \mathrm{~K}$ using the MFQ-AMP/S dynamical method and the DIM+SO interaction model: $E_{\text {phot }}=4.35 \mathrm{eV}-$ dashed line, $E_{\text {phot }}=4.50 \mathrm{eV}-$ solid line, and $E_{\text {phot }}=4.65 \mathrm{eV}-$ dotted line. For comparison, distributions obtained from MF calculations are also added as grey lines. The distributions are normalized so that for each photon energy they integrate up to relative abundances of the fragments. 
FIG. 1: See attachments.

FIG. 2: See attachments.

FIG. 3: See attachments. 
FIG. 4: See attachments.

FIG. 5: See attachments.

FIG. 6: See attachments.

FIG. 7: See attachments.

FIG. 8: See attachments.

FIG. 9: See attachments.

FIG. 10: See attachments.

FIG. 11: See attachments. 
TABLE I: Positive charge delocalization in the lowest fifteen electronic states of the two most stable isomers (see Fig. 1) of $\mathrm{Ar}_{5}^{+}$. Label 1 denotes the electronic ground state, labels 2 - 15 are used for the fourteen lowest electronically excited states of $\mathrm{Ar}_{5}^{+}$. Values given in parentheses correspond to the charges carried by the two atoms aside the linear core of the cluster which are neutral in the cluster electronic ground state. Excitations to states 2 - 14 contribute to the VIS absorption band (left-hand-side shoulder - states 2 and 3, main peak - states 4-12, and right-hand-side shoulder - states 13 and 14) while state 15 is responsible for the UV absorption band (see also Fig. 4b). Excitation energies, $E_{\text {exc }}$, are given in $\mathrm{eV}$, charges in per cent of the elementary charge. Note that the partial charges may sum up to $99-101 \%$ due to round off errors.

\begin{tabular}{ccccc}
\hline state & \multicolumn{3}{c}{ isomer1 } & \multicolumn{2}{c}{ isomer2 } \\
& $E_{\text {exc }}$ & charges & $E_{\text {exc }}$ & charges \\
\hline 1 & 0.00 & $20-54-(0,0)-27$ & 0.00 & $23-(0)-54-(0)-23$ \\
2 & 1.74 & $11-63-(5,5)-16$ & 1.73 & $12-(6)-64-(6)-12$ \\
3 & 1.85 & $10-62-(6,6)-16$ & 1.86 & $13-(6)-61-(6)-13$ \\
4 & 2.15 & $3-1-(28,28)-40$ & 2.19 & $21-(29)-0-(29)-21$ \\
5 & 2.22 & $2-0-(33,33)-32$ & 2.23 & $22-(28)-0-(22)-28$ \\
6 & 2.32 & $4-2-(41,41)-12$ & 2.30 & $5-(44)-2-(44)-5$ \\
7 & 2.44 & $47-1-(10,10)-32$ & 2.44 & $37-(12)-1-(12)-37$ \\
8 & 2.50 & $25-1-(35,35)-5$ & 2.49 & $22-(28)-0-(28)-22$ \\
9 & 2.58 & $32-1-(27,27)-13$ & 2.55 & $9-(40)-1-(40)-9$ \\
10 & 2.64 & $34-2-(26,26)-13$ & 2.60 & $36-(13)-2-(13)-36$ \\
11 & 2.72 & $18-1-(37,37)-7$ & 2.76 & $26-(22)-3-(22)-26$ \\
12 & 2.75 & $42-4-(20,20)-14$ & 2.78 & $9-(41)-0-(41)-9$ \\
13 & 3.10 & $16-33-(13,13)-25$ & 3.07 & $23-(11)-33-(11)-23$ \\
14 & 3.28 & $11-30-(18,18)-23$ & 3.30 & $16-(18)-32-(18)-16$ \\
15 & 4.55 & $25-46-(1,1)-28$ & 4.56 & $26-(1)-46-(1)-26$ \\
\hline
\end{tabular}


TABLE II: Time evolution of abundances $(f)$ of ionic trimer fragments after prolonging the integration time from $t=50 \mathrm{ps}$ to $t=200 \mathrm{ps}$ as obtained for $T=50 \mathrm{~K}$, the DIM+SO model, and selected photon energies $\left(E_{\text {phot }}\right)$. Note that all the ionic trimer fragments detected at the end of $50 \mathrm{ps}$ trajectories are unstable or metastable for the three photon energies considered and have enough energy to further dissociate. Values given in parentheses represent relative changes of the abundances of ionic trimer fragments during the time evolution from $t=50 \mathrm{ps}$ to $t=200 \mathrm{ps}$. Energies are given in $\mathrm{eV}$.

\begin{tabular}{ccccc}
\hline \multirow{2}{*}{$E_{\text {phot }}$} & \multicolumn{2}{c}{ MFQ-AMP/S } & \multicolumn{2}{c}{ MFQ-TFS/G } \\
& $f(50 \mathrm{ps})$ & $f(200 \mathrm{ps})$ & $f(50 \mathrm{ps})$ & $f(200 \mathrm{ps})$ \\
\hline 2.65 & 0.162 & $0.118(-27 \%)$ & 0.060 & $0.028(-53 \%)$ \\
2.85 & 0.352 & $0.266(-24 \%)$ & 0.144 & $0.084(-42 \%)$ \\
2.95 & 0.170 & $0.128(-25 \%)$ & 0.110 & $0.034(-69 \%)$ \\
\hline
\end{tabular}

TABLE III: Relative fractions $\left(\Delta f_{\mathrm{r}}\right)$ of unstable and/or metastable ionic dimer fragments detected at the end of $50 \mathrm{ps}$ trajectories and the time evolution of abundances $(f)$ of ionic dimer fragments after prolonging the integration time from $t=50 \mathrm{ps}$ to $t=200 \mathrm{ps}$ as obtained for $T=50 \mathrm{~K}$, the DIM+SO model, and selected photon energies $\left(E_{\mathrm{phot}}\right)$. Values given in parentheses represent relative changes of the abundances of ionic dimer fragments during the time evolution from $t=$ $50 \mathrm{ps}$ to $t=200 \mathrm{ps}$ due to the dimer and trimer decay, respectively. Energies are given in $\mathrm{eV}$.

\begin{tabular}{lcccccc}
\hline \multirow{2}{*}{$E_{\text {phot }}$} & \multicolumn{3}{c}{ MFQ-AMP/S } & \multicolumn{3}{c}{ MFQ-TFS/G } \\
& $\Delta f_{\mathrm{r}}(50 \mathrm{ps})$ & $f(50 \mathrm{ps})$ & $f(200 \mathrm{ps})$ & $\Delta f_{\mathrm{r}}(50 \mathrm{ps})$ & $f(50 \mathrm{ps})$ & $f(200 \mathrm{ps})$ \\
\hline 2.15 & $3 \%$ & 0.710 & $0.694(-2 /+0 \%)$ & $1 \%$ & 0.914 & $0.918(+0 /+0 \%)$ \\
2.35 & $6 \%$ & 0.334 & $0.318(-6 /+1 \%)$ & $1 \%$ & 0.540 & $0.548(+1 /+1 \%)$ \\
2.65 & $18 \%$ & 0.084 & $0.108(-2 /+31 \%)$ & $16 \%$ & 0.270 & $0.296(-3 /+13 \%)$ \\
2.85 & $76 \%$ & 0.110 & $0.150(-0 /+36 \%)$ & $60 \%$ & 0.252 & $0.278(-6 /+17 \%)$ \\
2.95 & $92 \%$ & 0.132 & $0.150(-3 /+17 \%)$ & $90 \%$ & 0.326 & $0.350(-10 /+17 \%)$ \\
\hline
\end{tabular}


TABLE IV: Relative abundances of the ionic photofragments produced in the UV region of the $\mathrm{Ar}_{5}^{+}$ photoabsorption spectrum as calculated for the DIM+SO model, $T=50 \mathrm{~K}$, and various dynamical approaches. Note that absolute majority of the dimer fragments obtained for the MFQ-AMP/S model (about 94-96\%) and all the dimer fragments obtained for the MFQ-TFS/G are metastable, more specifically, they are quasi-bound in electronically highly excited states, correlating asymptotically to ${ }^{2} P_{1 / 2}$ ionic monomers, and would probably dissociate via the radiative decay. The trimer fragments obtained for both MFQ approaches are unstable; they are produced in electronically excited states, correlating asymptotically to ${ }^{2} P_{1 / 2}$ ionic monomers, and have enough energy to dissociate via extending their trajectories to longer integration times.

\begin{tabular}{lllllllllll}
\hline $\begin{array}{l}E_{\text {phot }} \\
\text { (eV) }\end{array}$ & \multicolumn{2}{c}{$\mathrm{Ar}^{+}$} & $\mathrm{Ar}_{2}^{+}$ & $\mathrm{Ar}_{3}^{+}$ & $\mathrm{Ar}^{+}$ & $\mathrm{Ar}_{2}^{+}$ & $\mathrm{Ar}_{3}^{+}$ & $\mathrm{Ar}^{+}$ & $\mathrm{Ar}_{2}^{+}$ & $\mathrm{Ar}_{3}^{+}$ \\
\hline 4.35 & 0.951 & 0.049 & 0.001 & 0.946 & 0.052 & 0.000 & 0.932 & 0.066 & 0.002 \\
4.50 & 0.956 & 0.044 & 0.001 & 0.950 & 0.046 & 0.004 & 0.938 & 0.058 & 0.004 \\
4.65 & 0.957 & 0.041 & 0.002 & 0.962 & 0.034 & 0.004 & 0.920 & 0.070 & 0.010 \\
\hline
\end{tabular}

